\title{
Collective Thomson Scattering Study Using Gyrotron in LHD
}

\author{
Shin KUBO, Masaki NISHIURA, Kenji TANAKA, Takashi SHIMOZUMA, \\ Yoshinori TATEMATSU ${ }^{1)}$, Takashi NOTAKE ${ }^{1)}$, Teruo SAITO ${ }^{1)}$, \\ Yasuo YOSHIMURA, Hiroe IGAMI, Hiromi TAKAHASHI \\ and Namiko TAMURA ${ }^{2)}$ \\ National Institute for Fusion Science,322-6 Oroshi-cho, Toki 509-5292, Japan \\ ${ }^{1)}$ Research Center for Development of FIR Region, University of Fukui, Fukui 910-8507, Japan \\ ${ }^{2)}$ Department of Energy Science and Technology, Nagoya University, Nagoya 464-8463, Japan
}

(Received 16 January 2009 / Accepted 22 July 2009)

\begin{abstract}
The collective Thomson scattering (CTS) is one of the most promising methods for evaluating the ion velocity distribution function. The study of CTS diagnostic has been started utilizing the gyrotron and antenna/transmission systems installed in LHD for high power local electron heating. One of the high power gyrotrons at $77 \mathrm{GHz}$ is selected as a probing power source and a set of highly focused antenna system is used for the probing and receiving antenna. The specific feature of the system, receiver design are described and preliminary data obtained in for ECRH plasma in LHD are discussed.
\end{abstract}

(C) 2010 The Japan Society of Plasma Science and Nuclear Fusion Research

Keywords: collective Thomson scattering, ECRH, ECE, gyrotron, Gaussian beam, ion temperature

DOI: $10.1585 /$ pfr.5.S1038

\section{Introduction}

The direct and local measurement of the ion velocity distribution function is important in any fusion relevant plasma to study the behaviors of not only the bulk but also the high energy ions. The collective Thomson scattering (CTS) has long been attracted and intensively studied as one of the most promising diagnostic methods for the ion distribution function [1]. Frequency range required for the CTS in the fusion relevant plasma is determined from the collective scattering condition and scattering angle that gives the spatial resolution. This frequency range lies from infrared to millimeter wave [2-4]. Mainly due to a small scattering cross section, or scattering efficiency, CTS requires high power probe beam source with sharp single frequency spectrum and highly sensitive receiver near the frequency but avoiding a direct contamination of the probe frequency that requires a sophisticated stray suppression. The other important factor required for the spatially well resolved measurement of CTS is the well defined probe and receiving beams and their controllability. High power, sharp spectrum, and highly focussed well defined Gaussian beam are already realized for the electron cyclotron resonance heating $(\mathrm{ECRH})$ system using gyrotron and high power transmission/antenna in LHD. We have started the trial of CTS study utilizing the existing ECRH system in LHD [5] as well as that utilizing higher frequency $(400 \mathrm{GHz})$ gyrotron that is under development [6]. Expected necessary frequency range to deduce ion velocity distribution is $\pm 3 \mathrm{GHz}$ at the center frequency. In section 2 are discussed the characteristic feature of the

author's e-mail: kubo@LHD.nifs.ac.jp
ECRH system in LHD as a CTS probe and receive beam. Electron cyclotron emission (ECE) is expected to be the main source of noise for CTS diagnostic. The ECE spectrum expected at the line of receiving beam is discussed in section 3. Receiver system is described in section 4. Finally the preliminary data obtained with the system described here are shown in section 5.

\section{ECRH System as a Probe Beam and Receiver Antenna for CTS}

One of the newly installed $77 \mathrm{GHz}$ gyrotrons in LHD have achieved more than $1 \mathrm{MW}$ over $3 \mathrm{~s}$ [7]. The present ECRH system is illustrated in Fig. 1 a). Nine gyrotrons are in operational and eight corrugated transmission lines (6- 88.9 id and 2-31.75 $\mathrm{mm}$ id) are connected to LHD. Two sets of injection antenna are installed on each 4-LHD ports $(5.5 \mathrm{U}, 9.5 \mathrm{U}, 2-\mathrm{O}$ and $1.5 \mathrm{~L})$. The $9.5 \mathrm{U}$ port antenna set is selected as a CTS probe and receiving antenna, since $77 \mathrm{GHz}$ high power is available for the probing beam and the beam controllability is well established and confirmed [8]. This gyrotron has a triode gun that allows high frequency power modulation without degrading oscillating mode which is one of the important feature for the CTS. This gyrotron is connected to one of the Gaussian mirror antenna set. This antenna set includes one another Gaussian beam mirror suitable for receiving the scattered power from definite scattering volume. The injection antenna used was originally designed for $168 \mathrm{GHz}$. The beam evolutions for $77 \mathrm{GHz}$ is re-calculated using the same mirror sets and configurations as $168 \mathrm{GHz}$, using the mirror 


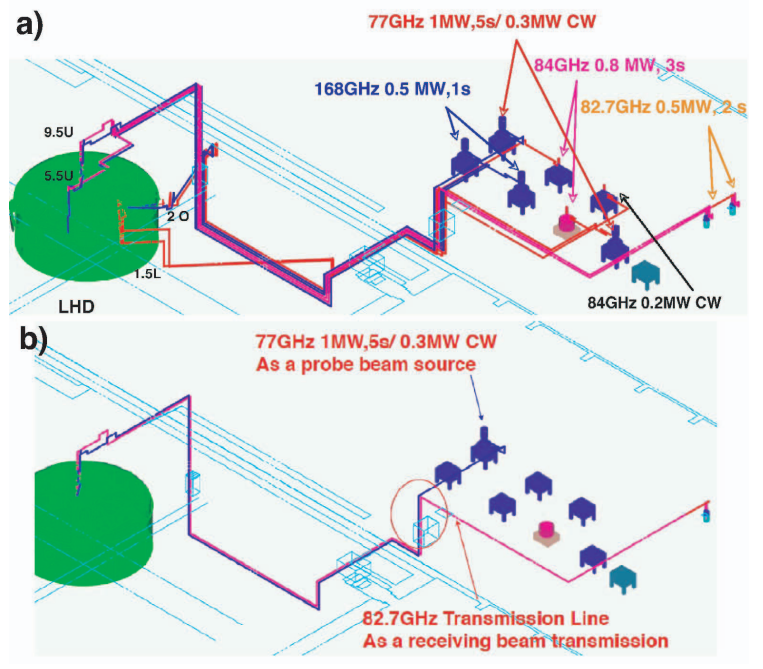

Fig. 1 a) Two sets of injection antenna system are installed on 4-ports of LHD, (5.5 U, 9.5 U, 1.5 L and 2 O). b) A set of transmission line/ antenna on the $9.5 \mathrm{U}$ port is selected for CTS. The set that $77 \mathrm{GHz} 1 \mathrm{MW}$ power is available is used for probe beam.

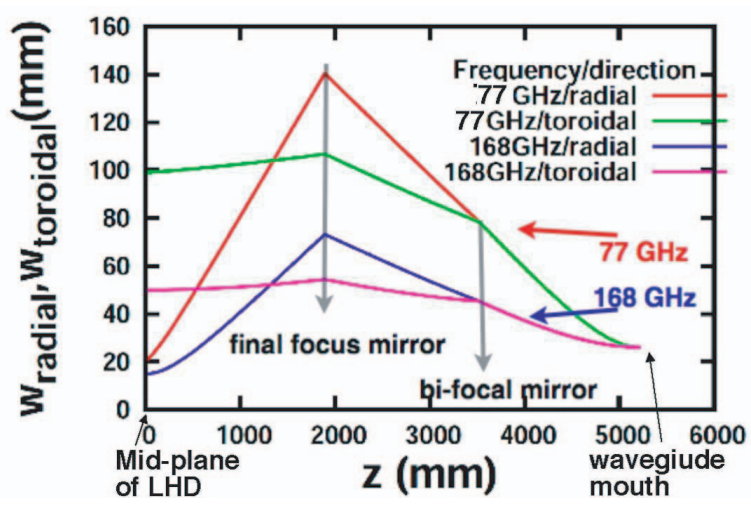

Fig. 2 Beam evolution at 9.5 U port antenna. Originally the focusing mirrors are optimized for $168 \mathrm{GHz}$ to focus the symmetric Gaussian beam radiated from $88.9 \mathrm{~mm}$ corrugated waveguide to an elliptical Gaussian beam on the mid-plane of LHD to have 1/e waist size of $15 \mathrm{~mm}$ and $50 \mathrm{~mm}$ in radial and toroidal directions, respectively. Recalculated beam evolutions for $77 \mathrm{GHz}$ are plotted. The waist size for $77 \mathrm{GHz}$ are also elliptical Gaussian with the waist size on the LHD-midplane 20 and $100 \mathrm{~mm}$.

curvature and phase plane transformation relation:

$$
\frac{\cos \phi}{\rho_{\sigma}}=\frac{1}{2 R_{\sigma, \text { in }}}+\frac{1}{2 R_{\sigma, \text { out }}} .
$$

Here, $\phi$ is the injection/reflection angle, $R_{\sigma, \text { in }}, R_{\sigma, \text { out }}$ and $\rho_{\sigma}$ are the phase radius of input and output beams and mirror curvature radius in $\sigma=$ toroidal or poloidal directions. Actual beam configuration is shown in Fig. 3 for the case where the center of the scattering volume lies near $\rho \approx 0.7$. The upper half of the flux surfaces of $\rho=0.1$ and 1.0 are also shown for reference. The effective scattering volume can be calculated by integrating over the space using the

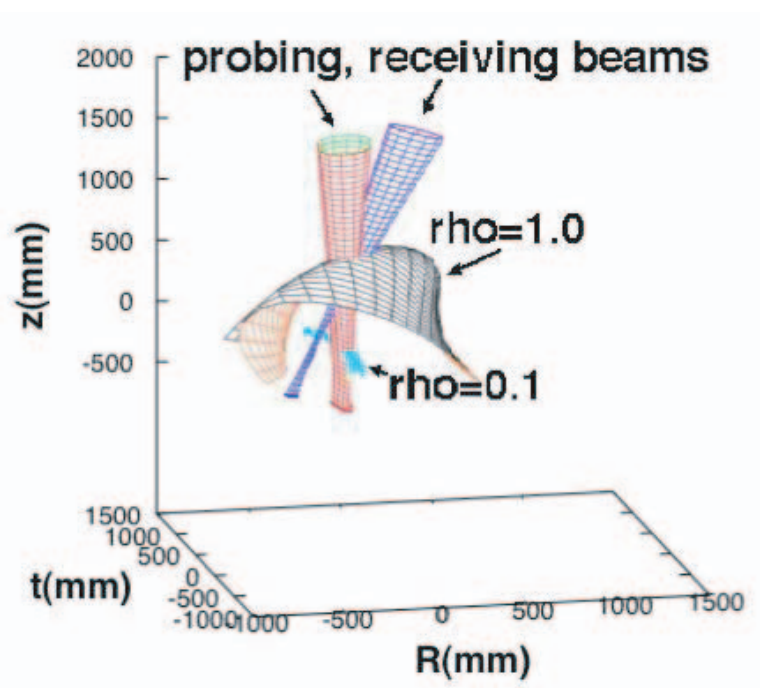

Fig. 3 3-D image of the probe and receiving beams.

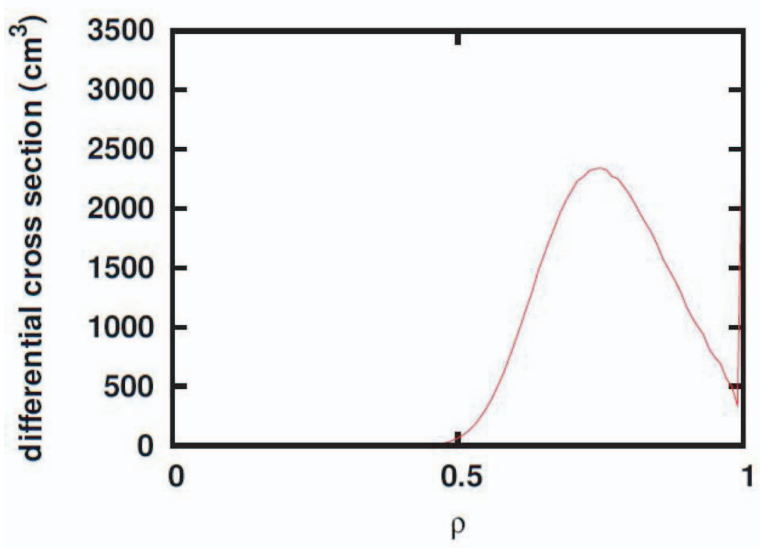

Fig. 4 Differential cross section of the scattering volume as a function of effective minor radius $\rho$. The scattering volume center is placed at $\mathrm{z}=0.5 \mathrm{~m}, \mathrm{R}=3.6 \mathrm{~m}$ on the vertically elongated poloidal cross section in LHD. Total effective scattering volume is about $700 \mathrm{~cm}^{3}$ in this case.

following formula

$$
\begin{aligned}
& \iint \exp \left(-\frac{x^{2}}{w_{0, \mathrm{x}}^{2}+\frac{\lambda^{2}\left(z-z_{0, \mathrm{x}}\right)^{2}}{\pi^{2} w_{0, \mathrm{x}}^{2}}}-\frac{y^{2}}{w_{0, \mathrm{y}}^{2}+\frac{\lambda^{2}\left(z-z_{0, \mathrm{y}}\right)^{2}}{\pi^{2} w_{0, \mathrm{y}}^{2}}}\right) \\
& \times \exp \left(-\frac{x^{\prime 2}}{w_{0, \mathrm{x}^{\prime}}^{2}+\frac{\lambda^{2}\left(z^{\prime}-z_{0, \mathrm{x}^{\prime}}\right)^{2}}{\pi^{2} w_{0, \mathrm{x}^{\prime}}^{2}}}-\frac{y^{\prime 2}}{w_{0, \mathrm{y}^{\prime}}^{2}+\frac{\lambda^{2}\left(z^{\prime}-z_{0, \mathrm{y}^{\prime}}\right)^{2}}{\pi^{2} w_{0, \mathrm{y}^{\prime}}^{2}}}\right) \\
& \quad \times \delta\left(\boldsymbol{r}^{\prime}-\overleftrightarrow{\boldsymbol{T}} \cdot \boldsymbol{r}\right) \mathrm{d} \boldsymbol{r} \mathrm{d} \boldsymbol{r}^{\prime} .
\end{aligned}
$$

Here, $\lambda$ is the wavelength, $w_{0, \sigma}, z_{0, \sigma}$ are the waist size and waist position in $\sigma=x, y, x^{\prime}, y^{\prime} .(x, y, z)$ is the local coordinate of injection beam and $\left(x^{\prime}, y^{\prime}, z^{\prime}\right)$ is that of receiving beam and $\stackrel{\leftrightarrow}{\boldsymbol{T}}$ is the conversion tensor of both local coordinates. The scattering volume for the case shown in Fig. 3 is about $700 \mathrm{~cm}^{3}$. This volume is distributed over the minor radius $0.78 \pm 0.2$ as shown in Fig. 4. This figure defines 
the spatial resolution of the CTS measurement using this antenna set.

\section{Background ECE as a Main Noise Source}

Main competing background noise source for CTS is the electron cyclotron emission (ECE). The background ECE level on the line of sight toward the receiver antenna should be estimated taking account of the actual antenna configuration. The calculation of the background ECE

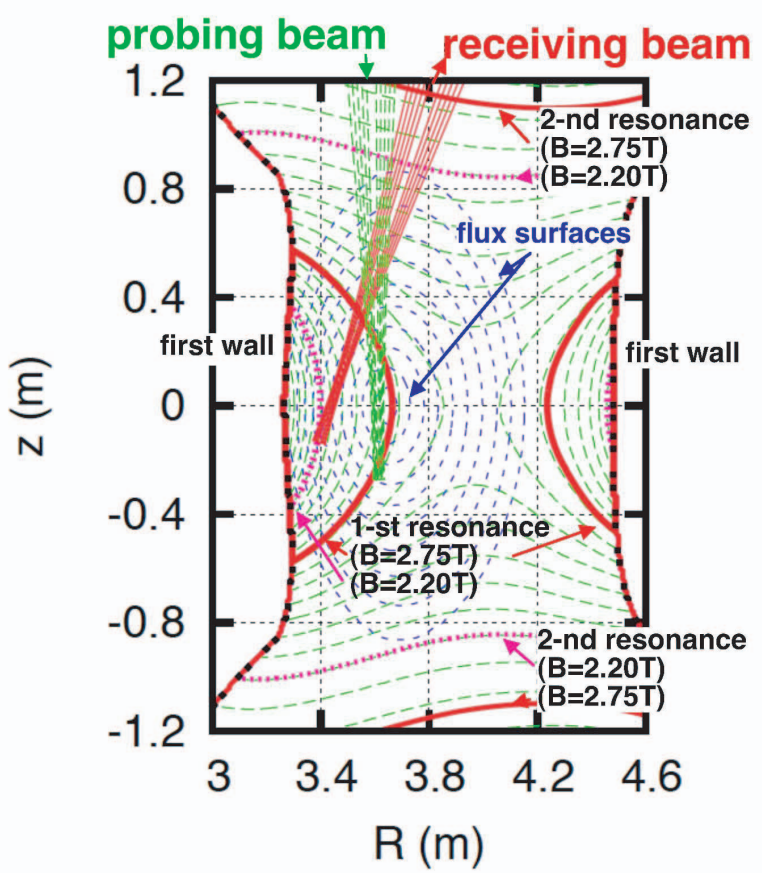

Fig. 5 Relation between probing, receiving beams, flux surfaces and EC resonances for the cases of magnetic field setting $\mathrm{B}_{0}=2.2$ and 2.75 Tesla on the vertically elongated cross section in LHD.

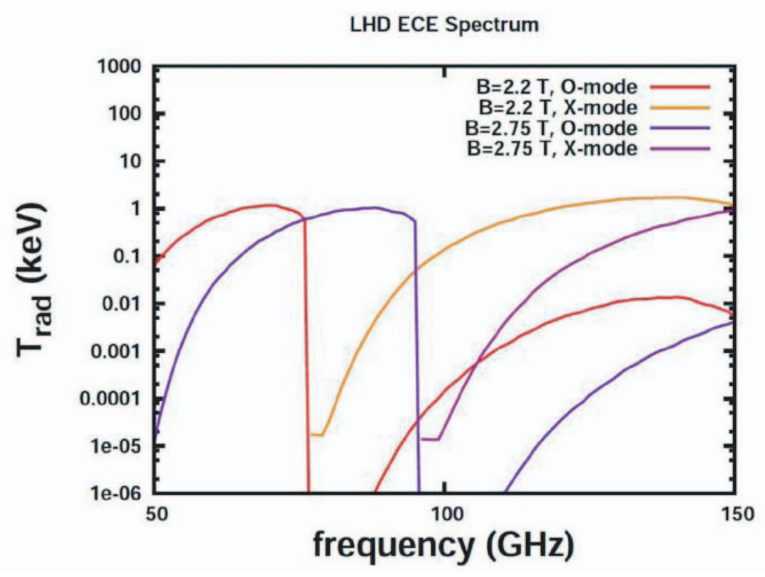

Fig. 6 Calculated ECE spectra for the line of sight of CTS receiving antenna. Electron temperature and density profile are assumed to be $3 \times\left(1-\rho^{2}\right)^{2}(\mathrm{keV})$, and $1 \times 10^{19}(1-$ $\left.\rho^{8}\right)^{2}\left(\mathrm{~m}^{-3}\right)$. Here are shown the spectra of $\mathrm{X}$ and $\mathrm{O}$ mode for the setting magnetic field $\mathrm{B}_{0}=2.2$ and $2.75 \mathrm{~T}$. spectrum is performed by solving the radiation transfer equation back along the line of sight. The method is described in Ref. [9]. For use of this $77 \mathrm{GHz}$ gyrotron as a probing beam, operational magnetic field should be selected so as to exclude the fundamental and second harmonic resonances of $77 \mathrm{GHz}$ on the line of sight inside the plasma confinement region, in order to reduce the ECE background that is considered to be the largest noise source. The relations between resonances and probing, receiving beam on the vertically elongated cross section in LHD are shown in Fig. 5. Setting higher magnetic field would place the fundamental resonance layer inner to the higher electron temperature region, causing higher background ECE level taking into account of the multireflection. On the other hand, setting lower magnetic field would place the second harmonic resonance layer on the receiving line of sight. In Fig. 6 are shown the calculated $\mathrm{O}$ and $\mathrm{X}$ mode ECE spectrum for the cases of central electron temperature $T_{\mathrm{e}, 0}=3 \mathrm{keV}$. By setting the magnetic field near 2.2 Tesla, one can expect the background ECE level minimum, although the multi-reflection effect can increase the contribution from fundamental resonance. Due to its complexity of magnetic field structure in LHD, there exists fundamental and second harmonic resonance simultaneously in any settings of magnetic field strength. In such configurations, some adjustment of setting magnetic field would necessary to minimize the ECE background and at the same time to avoid the absorption of scattered power. For the magnetic field setting at $2.75 \mathrm{~T}$, where the fundamental resonance layer crosses the magnetic axis, the intensity of the fundamental O mode ECE approach thermal level but of off-axis due to the line of sight. The fundamental X mode ECE does not appear because of cut-off.

\section{Receiver System for CTS}

A receiver system is installed on the upstream of the transmission line, which is normally used for high power transmission line for ECRH. A waveguide switch is attached on the $88.9 \mathrm{~mm}$ id corrugated waveguide transmission system at the marked position in Fig. $1 \mathrm{~b}$ ). A heterodyne receiver is placed at the output of the waveguide switch. The receiver consists of a high sensitive heterodyne radiometer. The circuit diagram is shown in Fig. 7 a).

At the front end, two multi-stage notch filters with the $3 \mathrm{~dB}$ band width of $300 \mathrm{MHz}$ and attenuation $-120 \mathrm{~dB}$ at the center frequency, $76.95 \mathrm{GHz}$, are placed to avoid the high level stray radiation that can damage the mixer or make ghost signal at the mixer and make saturation of the intermediate frequency (IF) amplifier. A pin-switch is also inserted to block the spurious mode which might be excited at the turn on or off of the gyrotron out of the notched but sensitive frequency. Band pass filter from 72 to $82 \mathrm{GHz}$ to filter out the lower side band of the mixer of the local frequency at $74 \mathrm{GHz}$ is also placed in front of the mixer. Intermediate frequency from $300 \mathrm{MHz}$ to $10 \mathrm{GHz}$ at the upper 


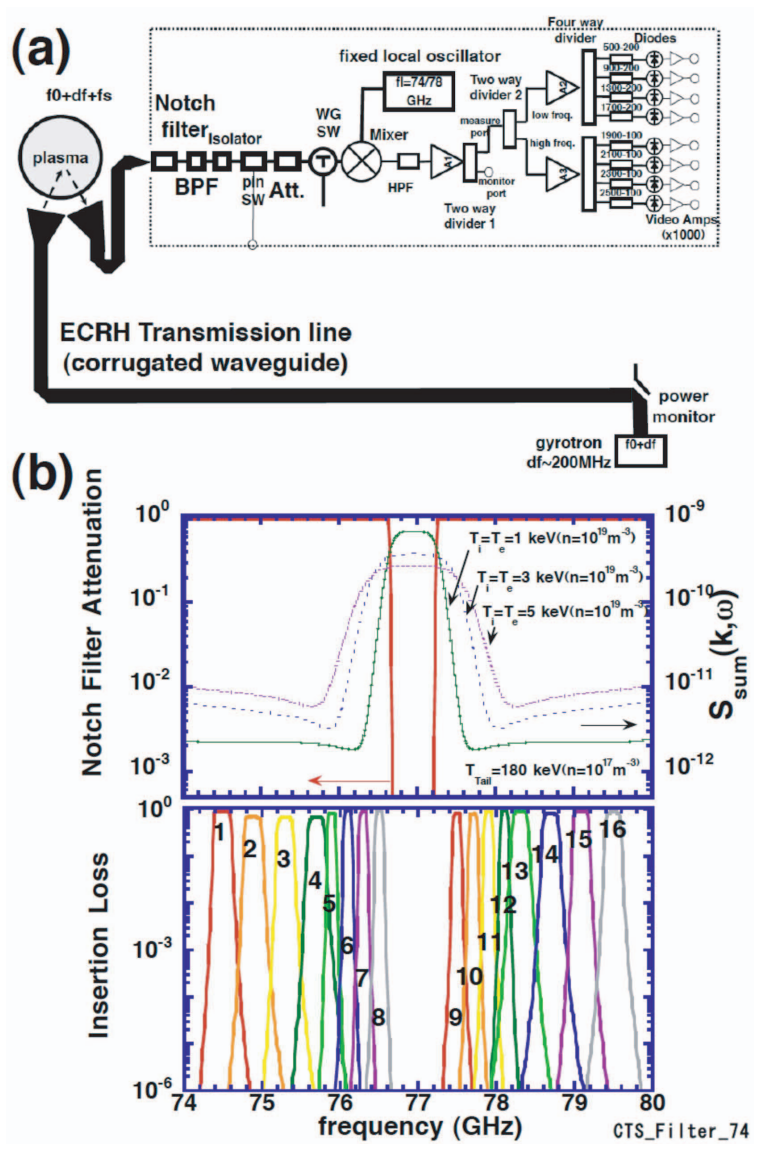

Fig. 7 (a) Block diagram of the heterodyne receiver for CTS in LHD. The notch filter, band pass filter and pin-switch are placed in front of the mixer. Fixed local frequency at $74 \mathrm{GHz}$ is normally used. (b) Characteristics of the filter prepared for the local frequency at $74 \mathrm{GHz}$ expected from the characteristic curve at the IF. Expected notch filter response with calculated CTS spectrum for $T_{\mathrm{e}}=T_{\mathrm{i}}=$ $1,3,5 \mathrm{keV}$ at the density of $1 \times 10^{19} \mathrm{~m}^{-3}$ with tail ions of $T_{\text {Tail }}=180 \mathrm{keV}, n_{\text {Tail }}=1 \times 10^{17} \mathrm{~m}^{-3}$ are shown in the upper column.

side band of the mixer is amplified by low noise amplifier and splitted to filter bank. Since the gyrotron oscillating frequency can subject to the shift of the order of $100 \mathrm{MHz}$ during the oscillation or at the ramping up phase of the anode voltage, IF center frequency tracking system using harmonic mixer will be also attached for the precise estimation of the bulk component. Filter bank consists of 8 to 16 filters at the first trial. Fig. 7 b) are shown the expected response of the notch and bank filters in the upper and lower column, respectively. Here, calculated CTS spectrum for the cases where the bulk ion and electron temperatures are 1,3 and $5 \mathrm{keV}$ with high enegy ions of $180 \mathrm{keV}$ are over plotted in the upper column. Primary noise sources other than the background ECE of the receiver system are from the mixer and amplifier.

\section{Preliminary Results}

At the adjustment phase of the CTS receiver system, the local oscillator was damaged and the Gunn oscillator

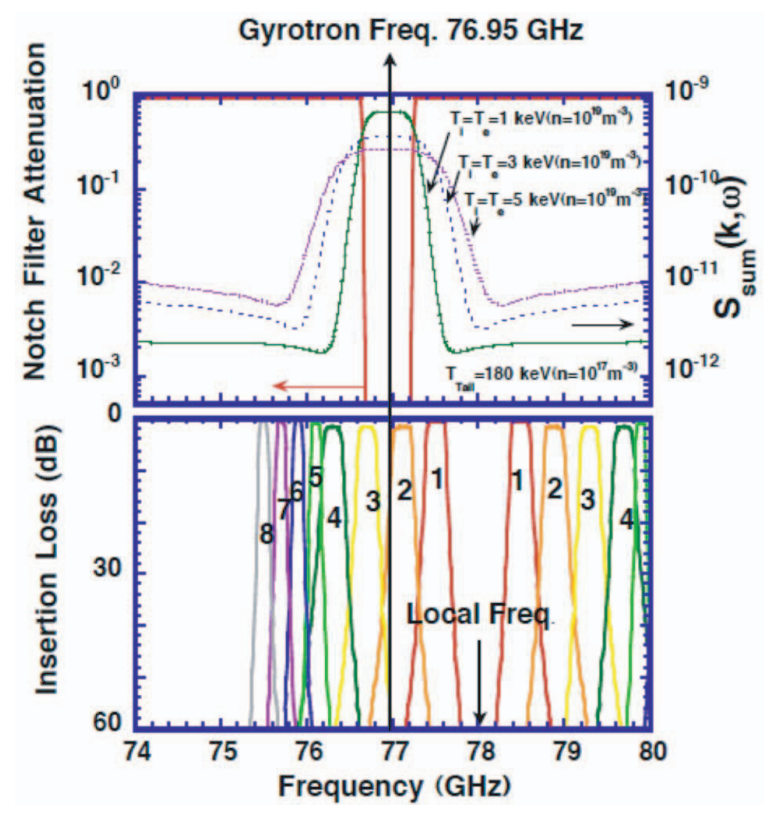

Fig. 8 Characteristics of the filter prepared for the local frequency at $78 \mathrm{GHz}$ expected from the characteristic curve at the IF. Expected notch filter response and CTS spectrum are shown above as Fig. 7.

at $78 \mathrm{GHz}$ is used in stead of $74 \mathrm{GHz}$. With this local frequency, expected frequency response of the receiver is shifted as shown in Fig. 8. In this case, channel 1 to 4 becomes sensitive at both upper and lower side bands of the local frequency. As is expected from the calculated CTS spectrum, channel 1 to 4 are sensitive to the bulk ion temperature and channel 5 to 8 are more sensitive to high energy ion components. Preliminary signals were obtained from the ECRH plasma without NBI at the magnetic field of 2.75 Tesla. Electron density levels was $1 \times 10^{19} \mathrm{~m}^{-3}$. Plasma parameters are kept only by the $77 \mathrm{GHz}$ ECRH which is also used as a probing beam. Nominal electron temperature was $3 \mathrm{keV}$ at the center. The injected power is $100 \%$ modulated at the frequency of $50 \mathrm{~Hz}$ from 0.25 to $1.15 \mathrm{~s}, 1.25$ to $1.75 \mathrm{~s}$ and $1.85 \mathrm{~s}$ to $2.25 \mathrm{~s}$. In Fig. 9 are shown detected signals at each filer channel from 1 to 8 indicated in Fig. 8. Raw signals contain sharp spikes at each modulation that may be attributed to the spurious mode oscillation from the gyrotron. These spikes are almost subtracted but failed from 1.3 to $1.6 \mathrm{~s}$ in this figure. The signals during on and off phases are separated and displayed by red and green lines. It seems that the background ECE level and scattered signal level are well separated at channels 1 to 4 and barely at channel 5 and 6. Shown in Fig. 10 are the expanded signals in time from 1.0 to $1.1 \mathrm{~s}$. These signals show clear characteristics of the scattering signals in contrast to the change of the ECE background which is small and should be continuous. It should be noted that channel 2 and 3 are still sensitive to the back ground ECE at the upper side-band, although almost the lower sidebands are within notched frequency range as indicated in Fig. 8. Although several checks are to be done, these sig- 


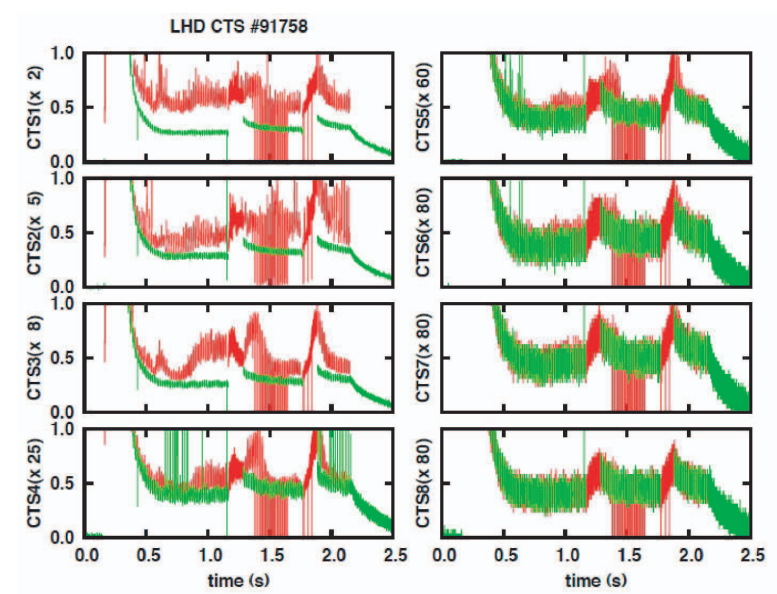

Fig. 9 Preliminary data from each filter bank channel as indicated in Fig. 8. Detected signals are separated by on and off phase of the probing beam and indicated by red and green lines.

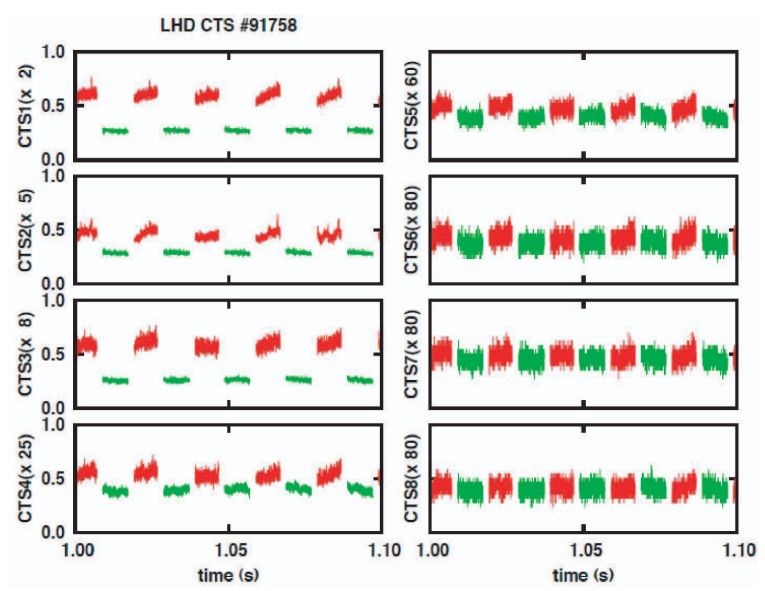

Fig. 10 Expanded figure in time from 1.0 to $1.1 \mathrm{~s}$ of Fig. 9. The increase of the ECE background can well be separated from the step-wise increase/decrease at turn-on and -off time especially in channels 1 to 6 .

nals show expected feature of the scattering signals. The sensitivity calibration of each channels are underway. The deduction of the CTS spectrum from these data would be possible after this calibration.

\section{Summary}

The CTS system is conformed utilizing the characteristic features of the LHD ECRH system. These features in- cludes well controlled and strongly focused Gaussian beam antenna, high power low loss transmission lines. From the expected Gaussian beam, the method of estimating the beam cross section is established and shown the spatial resolution of CTS measurement. CTS receiver is designed and constructed to detect the scattering signals in the range of $\pm 3 \mathrm{GHz}$ at $77 \mathrm{GHz}$. Preliminary signals are obtained for ECRH plasma at the density of $1 \times 10^{19} \mathrm{~m}^{-3}$. Furthermore check and calibration is necessary to confirm and deduce ion velocity distribution function from these data.

\section{Acknowledgement}

This work has been supported by the National Institute for Fusion Science under NIFS08ULRR501, 503 and also partly by the Grant in Aid for Scientific Research on Priority Areas, KAKENHI 20026009.

[1] H. Bindslev, S. K. Nielsen, L. Porte, J. A. Hoekzema, S. B. Korsholm, F. Meo, P. K. Michelsen, S. Michelsen, J. W. Oosterbeek, E. L. Tsakadze, E. Westerhof and P. Woskov, Phys. Rev. Lett. 97, 205005 (2006).

[2] T. Kondoh, S. Lee, D. P. Huchinson and R. K. Richards, Rev. Sci. Instrum. 72, 1143 (2001).

[3] S. Michelsen, S. B. Korsholm, H. Bindslev, F. Meo, P. K. Michelsen, E. L. Tsakadze, J. Egedal, P. Woskov, J. A. Hoekzema, F. Leuterer and E. Westerhof, Rev. Sci. Instrum. 75, 3634 (2004).

[4] F. Meo, H. Bindslev, S. B. Kolsholm, E. L. Tsakadze, C. I. Walker, P. Woskov and G. Vayakis, Rev. Sci. Instrum. 75, 3585 (2004).

[5] M. Nishiura, K. Tanaka, S. Kubo, T. Saito, Y. Tatematsu, T. Notake, K. Kawahata, T. Shimozuma and T. Mutoh, Rev. Sci. Instrum. 79, 10E731 (2008).

[6] T. Notake, T. Saito, Y. Tatematsu, S. Kubo, T. Shimozuma, K. Tanaka, M. Nishiura, A. Fujii, La Agusu, I. Ogawa and T. Idehara, Rev. Sci. Instrum. 79, 10E732 (2008).

[7] H. Takahashi, T. Shimozuma, S. Kubo, S. Ito, S. Kobayashi, Y. Yoshimura, H. Igami, Y. Mizuno, Y. Takita, T. Mutoh, T. Kariya, R. Minami and T. Imai, Submitted to Fusion Science and Technology.

[8] S. Kubo, T. Shimozuma, Y. Yoshimura, H. Igami, H. Takahashi et al., Proc. 22nd IAEA Fusion Energy Conference, 13-18 October 2008 Geneva, Switzerland. EX/P6-14.

[9] S. Kubo, H. Igami, Y. Nagayama, S. Muto, T. Shimozuma, Y. Yoshimura, H. Takahashi and T. Notake, Proc. of International Congress on Plasma Physics 2008, 8-12 September 2008, Fukuoka Japan; Submitted to Plasma Fusion Res. 